intake as a risk factor for fracture. Osteoporos Int 2005; $16: 737-42$.

4. Kanis JA, Johnell O, Oden A, et al. Smoking and fracture risk: a meta-analysis. Osteoporos Int 2005; 16:155-62.

5. Raghunath AS, Hungin AP, Mason J, et al. Symptoms in patients on long-term proton pump inhibitors: prevalence and predictors. Aliment Pharmacol Ther 2009;29:431-9.

6. El-Serag $\mathrm{H}$. The association between obesity and GERD: a review of the epidemiological evidence. Dig Dis Sci 2008;53:2307-12.

DOI:10.1503/cmaj.1090002

\section{Apology laws}

In their editorial on medical errors and apology laws, MacDonald and Attaran stated that when a medical error has occurred, patients and their families seek disclosure, apology and restitution. ${ }^{1}$ We must be clear, however, about what is meant by the term "medical error." I and others believe that the term should be reserved for acts of omission or commission that would have been judged wrong by similarly trained peers who had the same information (at the same time) as the person responsible for the act. Banja ${ }^{2}$ argues that the use of this substitution test reduces the natural tendency to hindsight bias in determining preventability.

Apology laws seem chiefly concerned with protecting admissions of liability. I believe that it is better to immediately express sympathy, discuss the facts and promise investigation, with subsequent further disclosure after internal or external review has determined the most likely causes of the situation.

I do not doubt that it would help a plaintiff's legal case if self-incriminating statements by the patient's physician were admissible in court proceedings, but why is there a court proceeding at all in such situations? If physicians or institutions thoughtfully admit an error (as defined above) that caused harm, then they should encourage their liability insurer to negotiate reasonable compensation without requiring their patient to sue them. If the patient's adverse outcome or event could not normally have been prevented because of the inherent uncertainty in medicine, then the physician and institution should help the patient to understand and accept this conclusion as part of the disclosure and healing process (and should vigorously defend in court the care they provided, if necessary). In either situation, attempting to take back or protect the physician's initial statements seems a somewhat contorted strategy for pursuing the resolution we seek.

\section{Daniel O'Connell PhD}

Institute for Healthcare Communication, Seattle, Wash.

Competing interests: Daniel O'Connell is a consultant to the Institute for Healthcare Communication.

\section{REFERENCES}

1. MacDonald N, Attaran A. Medical errors, apologies and apology laws [editorial]. CMAJ 2009; 180:11.

2. Banja JD. Medical errors and medical narcissism Sudbury (MD): Jones and Bartlett Publishers; 2005

DOI:10.1503/cmaj.1090007

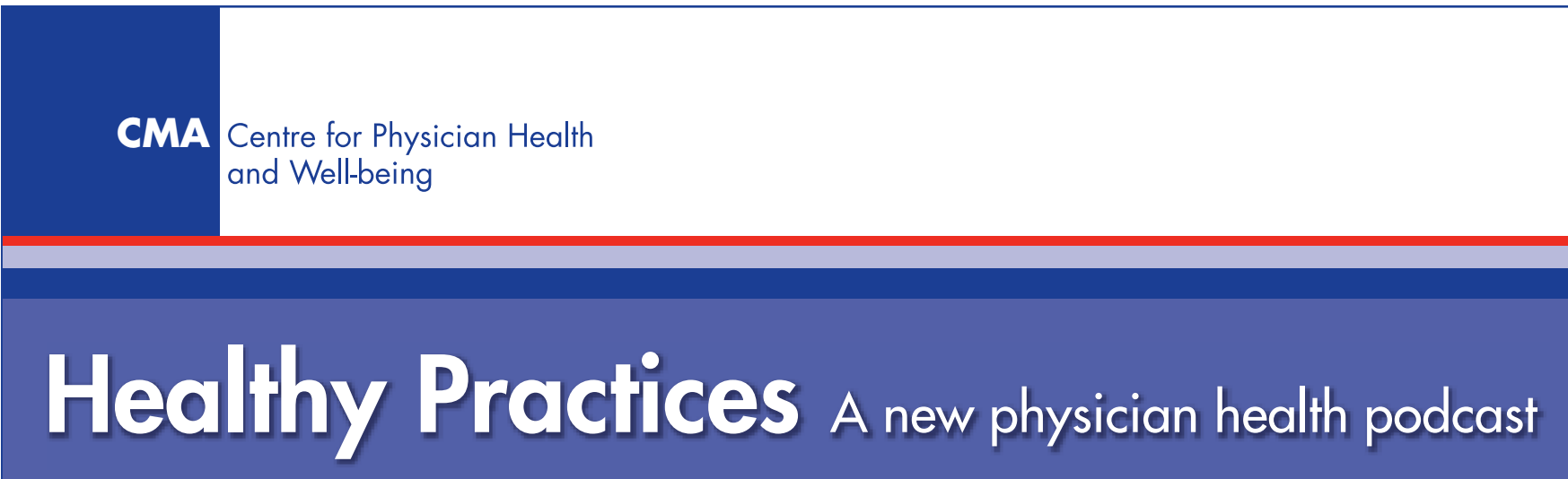
Hosted by: Dr. Mamta Gautam, the "Doctor's Doctor"

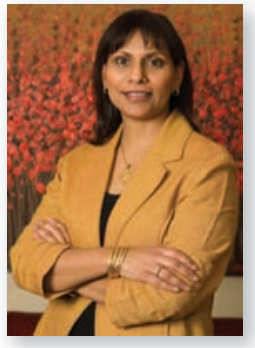

- Explores work-life challenges and solutions

- 5 new episodes: What makes a physician healthy? 